\title{
Removing Partial Occlusion from Blurred Thin Occluders
}

\author{
Scott McCloskey, Michael Langer, and Kaleem Siddiqi \\ Centre for Intelligent Machines, McGill University \\ $\{$ scott, langer, siddiqi\}@cim.mcgill.ca
}

\begin{abstract}
We present a method to remove partial occlusion that arises from out-of-focus thin foreground occluders such as wires, branches, or a fence. Such partial occlusion causes the irradiance at a pixel to be a weighted sum of the radiances of a blurred foreground occluder and that of the background. The result is that the background component has lower contrast than it would if seen without the occluder. In order to remove the contribution of the foreground in such regions, we characterize the position and size of the occluder in a narrow aperture image. In subsequent images with wider apertures, we use this characterization to remove the contribution of the foreground, thereby restoring contrast in the background. We demonstrate our method on real camera images without assuming that the background is static.
\end{abstract}

\section{Introduction}

When out of focus, an object's image is well described by the convolution of the sharply-focused image of the object with the point spread function of the lens. Near discontinuities in depth, however, the situation becomes more complicated [1]. When focused on the background, a finite aperture lens projects light from both foreground (occluding) and background (occluded) objects to the same point on a sensor. Such points are partially occluded in that the irradiance is due to both the foreground and background objects. While we have previously addressed partial occlusion adjacent to regions of complete occlusion [10], this work describes an extension to handle regions of partial occlusion without corresponding regions of complete occlusion. This latter cases arises when the occluding object is too thin relative to the aperture to give rise to complete occlusion, as in the case of a metal grating.

Such configurations arise in surveillance, for example, when a camera captures images through a fence or other sparse occluder. Using a real (i.e., non-pinhole) aperture, the camera's lens cannot sharply render both

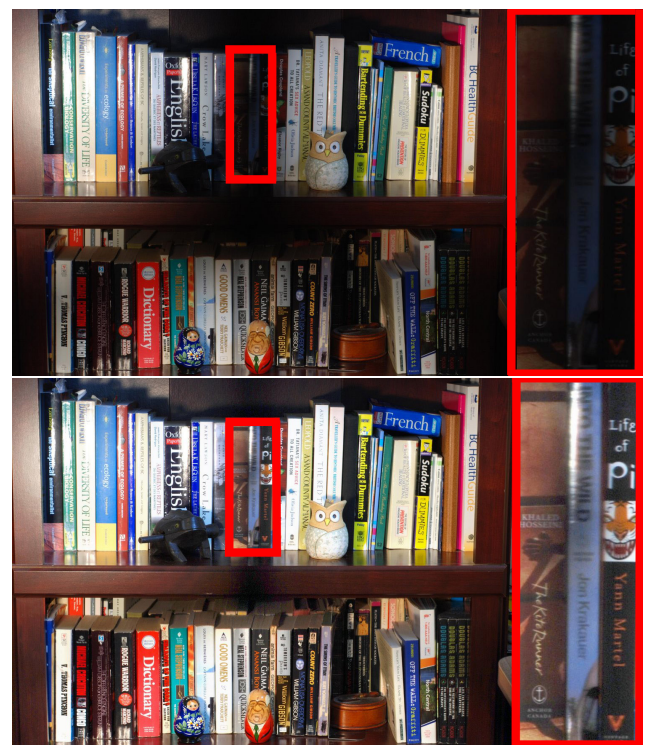

Figure 1. (Top) Image with partial occlusion, taken through a metal grating. (Bottom) The background, as estimated by our algorithm. Red insets show detail.

the background and the fence in the same image. Assuming, as we do, that the lens is focused on the background, the foreground will appear defocused and obscure details of the background, as shown in Fig. 1 (top). Our objective is to remove the contribution of the defocused foreground, in order to clarify details on the background, as shown in the example output in Fig. 1 (bottom).

Our assumptions are as follows. First, we assume that the foreground is approximately fronto-parallel, and that it lacks high-contrast texture. Second, we assume that the camera's focus and capture settings are known and are chosen to properly expose the background. Provided that it is sufficiently far from the foreground occluder, we do not assume any particular background geometry. Finally, we assume the camera is in a fixed position relative to the foreground, so we can characterize the occluder's position and size. 

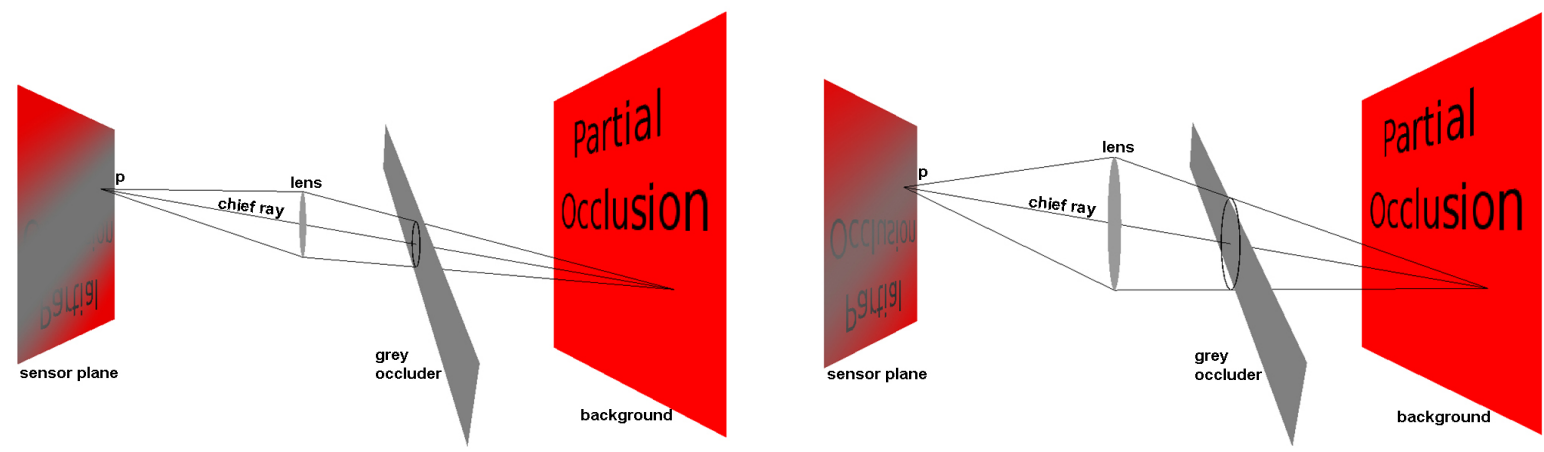

Figure 2. Imaging a red scene object behind a grey occluding object. These figures illustrate qualitative aspects of our model, such as the cone in the scene space and a point $p$ that is completely occluded through a narrow aperture (left image) but only partially occluded through a wider aperture (right image).

\section{Related Work}

Defocus blur has been well-studied in the computer vision literature, particularly as it relates to $3 \mathrm{D}$ reconstruction $[12,3]$. Much of the early literature is based on the shift-invariant model of blur, which is only applicable in regions of an image corresponding to equiblur surfaces. Like our method, others have recently addressed background restoration near blurred edges: Bhasin and Chaudhuri [2], Favaro and Soatto [6], and Hasinoff and Kutulakos[7]. While each of these methods can perform background estimation, they do so as part of a full scene reconstruction that includes its 3D shape. In order to make this larger problem tractable, they each assume access to several images of the same static scene, each image having been taken with different camera settings. Because the static scene assumption does not hold applications with moving backgrounds, e.g. surveillance, we make the weaker assumption that the occluding foreground and the camera remain in fixed positions relative to one another; the background is free to vary with time.

Though we model partial occlusion using the image matting equation, our method differs from matting techniques in fundamental ways. First, whereas matting methods attempt to extract the foreground in order to produce a composite with a novel, well-captured background, our objective is to discard the foreground in order to reconstruct the whole background. Second, whereas matting methods assume that $\alpha$ is 0 or 1 on all but a small number of pixels, we assume that there are many pixels with $0<\alpha<1$ and few (if any) pixels with $\alpha=1$. Third, we assume minimal input from the user (as little as a single click), whereas matting methods assume much richer (and time-consuming) input in the form of trimaps [4, 13] or scribbles [9]. Fourthly, our method is quite different from matting in that it is built on an explicit model of image formation. Though effective when applied in their traditional domain, Dai and $\mathrm{Wu}$ [5] have demonstrated that matting methods perform poorly in regions of partial occlusion.

A number of related methods have been presented recently that recover background intensity in regions of partial occlusion. With respect to motion blur, Dai and $\mathrm{Wu}$ [5] and Jia [8] have both demonstrated de-blurring methods that assume users manually delineate the foreground and background regions. In both these methods, as in our previous work [10,11], it is assumed that there is some region of the image in which only the foreground is present. This is not the case in the images that we process in the current paper, e.g. Fig. 1 (top).

In the special case where the foreground contributes negligible exposure to the image, partial occlusion is closely related to image vignetting. Existing methods, e.g. [14], which invert the intensity reduction at image edges due to attenuation by the lens, cannot be applied to the attenuation due to an object in the scene. Vignetting methods attempt to fit a global model in which the attenuation is symmetric about the lens' center of projection, whereas we estimate a local model of an occluder that is outside of the camera body and that can have a more general shape.

\section{Occlusion Model}

As in image matting, we model the average radiance $R$ incident at pixel $p$ as:

$$
R(p)=\alpha(p) R_{f}(p)+(1-\alpha(p)) R_{b}(p),
$$

where $R_{f}$ is the radiance of the foreground, $R_{b}$ is the radiance of the background, and $\alpha$ denotes the relative contribution of the two. With this notation, the image irradiance is $R(p)$ times the solid angle of the lens aperture. Because we assume that the foreground is severely defocused, we consider the special case in which the foreground's contribution is constant, i.e., $R_{f}\left(p_{1}\right)=R_{f}\left(p_{2}\right) \forall p_{1}, p_{2}$, and drop the indexing of $R_{f}$. 
This model applies to each color plane in an image, i.e., the $R_{f}$ and $R_{b}(p)$ are RGB triplets but $\alpha$ is the same in each color plane since it arises from scene geometry.

The crux of our problem, then, is the estimation of $\alpha$ at all points in the image, and of the global $R_{f}$. We use the reverse projection blur model of Asada et al [1], as illustrated in Fig. 2. The opacity $\alpha(p)$ takes a value of 0 when the cone centered on $p$ 's chief ray doesn't intersect the occluding object, and it takes the value 1 when complete occlusion occurs, i.e., when the occluding object spans the solid angle of the cone. At pixels with partial occlusion, $\alpha(p)$ is modelled as follows. Let $\chi(p)$ be the indicator function which takes a value of 1 when the chief ray strikes the foreground, and is 0 elsewhere. For each $p$, there is a blur disk $D$ centered at $p$, which accounts for the blur of the foreground occluder. By the thin lens law, this blur disk is a pillbox function of constant width (diameter) $w$, where $w$ is determined by the constant depth $d_{o}$ of the occluder and by the focal length $f$, aperture $F$, and distance to the lens $d_{s}$ as

$$
w=\frac{f}{F}\left|1-d_{s}\left(\frac{1}{f}-\frac{1}{d_{o}}\right)\right| .
$$

Since the blur disks all have the same width $w$, we can interpret $D$ as a blur kernel in a convolution:

$$
\alpha(p)=D * \chi(p) .
$$

Since $\chi(p)$ takes values either 1 or 0 when the chief ray strikes the foreground or background, respectively, it follows that $\alpha(p)$ is the fraction of the area of the blur disk centered at $p$ that is covered by the occluder. Given this relationship, the problem of estimating $\alpha$ is reduced to estimating $\chi$ and $w$.

\section{Images without Complete Occlusion}

In our previous work [11], we have shown that $R_{f}$, $\chi$, and $w$ can be estimated with minimal user effort in the case where the foreground object is thicker than $w$, in which case regions of complete occlusion $(\alpha=1)$ are found in the image. In the absence of such regions, however, neither our method nor the methods of $[5,8]$ can be used, since there is no foreground region for a user to delimit. In order to handle such cases, we extend our method to use information from two images. Suppose we are given one image - taken with a relatively small aperture, as in Fig. 2 (left) - with regions of complete occlusion, from which we estimate the occluder function $\chi$ and the blur width $w$ using our existing method [11]. Suppose further that we are given a second image which has been captured with a larger aperture, as in Fig. 2 (right). Knowing the aperture values $F_{1}$ and $F_{2}$ of both images (commonly stored in the

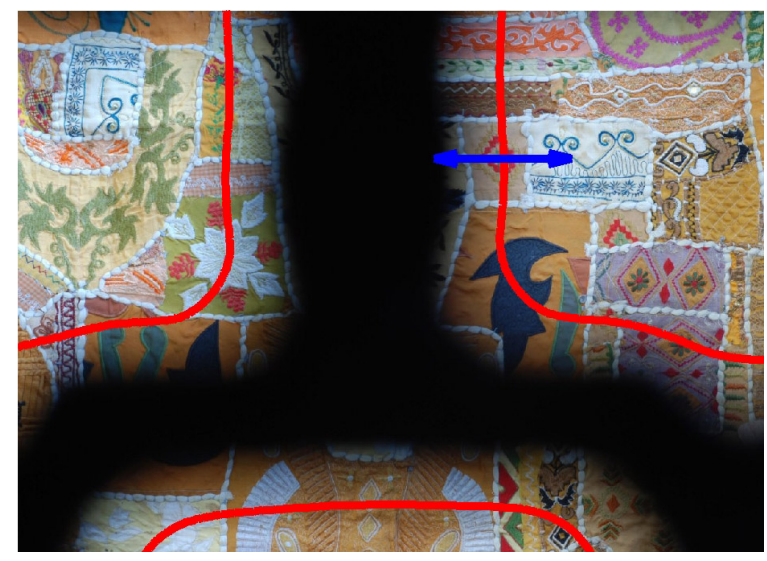

Figure 3. Image of a mural, taken with a relatively small (f/11) aperture. The foreground is the same as in Fig. 1, a metal grating. Our existing method [11] estimates the occluder function $\chi$ (area within red contours has $\chi=1$ ) and blur width $w$ (length of blue arrow) for this configuration.

EXIF header data of JPEG images), the blur width in the larger aperture image can be calculated using Eq. 2. Specifically, the blur width in the second (wide aperture) image is computed from the estimated blur width $w_{1}$ from the first (small aperture) image via

$$
w_{2}=\frac{F_{1}}{F_{2}} w_{1} .
$$

Provided that the occluding object is in a fixed position relative to the camera, the foreground indicator function $\chi$ has not changed, and so the $\alpha$ function corresponding to the second image can be found by Eq. 3 using $\chi$ and a blur disk of diameter $w_{2}$.

Given an estimate of $\alpha$ and $R_{f}$, then, we can estimate the background intensity

$$
\widehat{R_{b}}(p)=\frac{R(p)-\alpha(p) R_{f}}{(1-\alpha(p))} .
$$

When the foreground is severely under-exposed, the second term in the numerator vanishes and this processing implements a space-varying contrast enhancement.

\section{Demonstration on Real Images}

Figs. 1 and 3 illustrate our method. A smaller aperture $(f / 11)$ image (Fig. 3$)$ is used to establish the foreground occluder function $\chi$ and the blur width $w_{f / 11}$ for this setting. The wide aperture $(f / 5)$ image (Fig. 1 top) is processed using $\chi$ and $w_{f / 5}=\frac{11}{5} w_{f / 11}$ to produce the result image shown in Fig. 1 (bottom), in which background details are restored to their proper contrast. In addition to using a different aperture for the second 

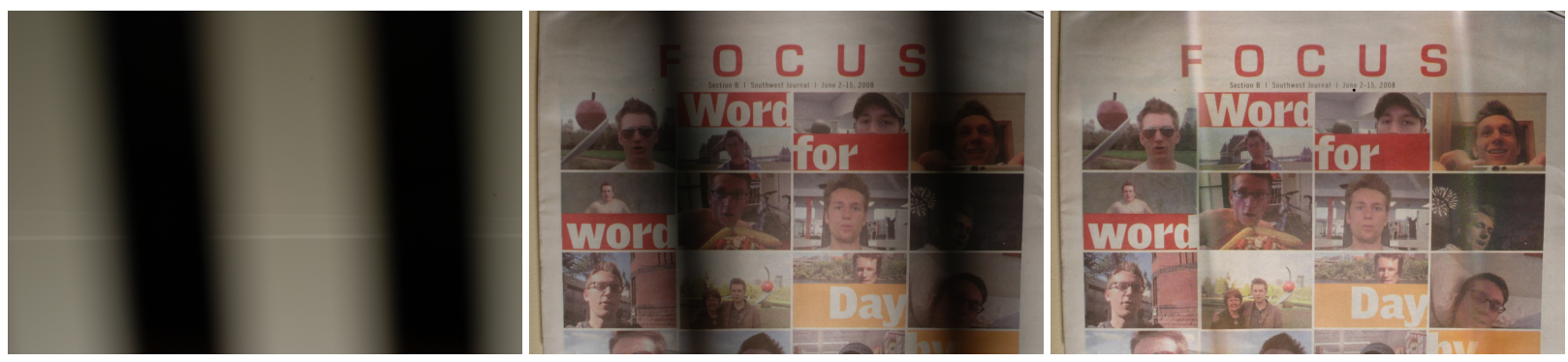

Figure 4. (Left) Narrow aperture (f/11) image of a white background, taken through a metal grating. (Center) Image of a newspaper, taken with a wider (f/6.3) aperture. Our method estimates the background without occlusion (Right).

image, note that the background has changed, the mural having been replaced by a bookcase.

Note also that the image in Fig. 1 (top) does not contain any completely occluded pixels, as the width of the blur disk $w_{2}$ corresponding to the aperture is now so great that it exceeds the width of the occluding object. In this case, processing the image according to eq. 5 with the $\alpha$ estimated from $w_{2}$ removes the contribution of the occluding object from the entire image. We emphasize that this two image method does not assume that the background scene is fixed, unlike other methods such as [6]. Moreover, unlike these multiple image methods, our method can handle specularities in the background region, as seen in the English dictionary shown in Fig. 1.

A second example is shown in Fig. 4, where the background is a newspaper page and the foreground is non-equifocal (the blur increases from bottom to top), violating one of our assumptions. In this case, though the true blur width varies throughout the image, our method still recovers detail on the background (right), albeit with minor artifacts. The narrow aperture image in this case (left) is taken against a blank background, illustrating how a calibration image can be used, e.g. when installing a surveillance camera.

\section{Conclusions}

We have described and demonstrated a method to remove partial occlusion due to foreground occluding objects in images that lack complete occlusion. As we have illustrated, this situation arises when images are captured through fences or other sparse occluders. We characterize the occluder's position and degree of blur in a narrow aperture image and use this information, together with the lens settings, to find the degree of blur and opacity in the wider aperture image. We make no assumption that the background scene is fixed, making this method applicable to real-world applications. In light of this new capability, the positioning of surveillance cameras need not be constrained in order to avoid such occlusions.

\section{References}

[1] N. Asada, H. Fujiwara, and T. Matsuyama. Seeing behind the scene: Analysis of photometric properties of occluding edges by the reversed projection blurring model. IEEE Trans. Pattern Anal. Machine Intell., 20(2):155-167, Feb. 1998.

[2] S. Bhasin and S. Chaudhuri. Depth from defocus in presence of partial self occlusion. In $I C C V$, pages I: 488-493, 2001.

[3] S. Chaudhuri and A. N. Rajagopalan. Depth from Defocus: A Real Aperture Imaging Approach. SpringerVerlag, 1998.

[4] Y. Y. Chuang, B. Curless, D. H. Salesin, and R. Szeliski. A bayesian approach to digital matting. In $C V P R$, pages II:264-271, 2001.

[5] S. Dai and Y. Wu. Removing partial blur in a single image. In CVPR, pages 2544-2551, 2009.

[6] P. Favaro and S. Soatto. Seeing beyond occlusions (and other marvels of a finite lens aperture). In $C V P R$, pages II: 579-586, 2003.

[7] S. Hasinoff and K. Kutulakos. A layer-based restoration framework for variable-aperture photography. In ICCV, pages 1-8, 2007.

[8] J. Jia. Single image motion deblurring using transparency. In $C V P R$, pages 1-8, 2007.

[9] A. Levin, D. Lischinski, and Y. Weiss. A closed-form solution to natural image matting. IEEE Trans. Pattern Anal. Machine Intell., 30(2):228-242, 2008.

[10] S. McCloskey, M. S. Langer, and K. Siddiqi. Seeing around occluding objects. In ICPR, pages 963-966. IEEE Computer Society, 2006.

[11] S. McCloskey, M. S. Langer, and K. Siddiqi. Automated removal of partial occlusion blur. In $A C C V$, pages I: 271-281, 2007.

[12] A. P. Pentland. A new sense for depth of field. IEEE Trans. Pattern Anal. Machine Intell., 9(4):523-531, July 1987.

[13] J. Sun, J. Jia, C.-K. Tang, and H.-Y. Shum. Poisson matting. ACM Transactions on Graphics, 23(3):315321, Aug. 2004.

[14] Y. Zheng, J. Yu, S. Lin, S. Kang, and C. Khambamettu. Single-image vignetting correction using radial gradient symmetry,. In $C V P R$, pages I: $1-8,2008$. 